\title{
CHILDHOOD OBESITY, RENAL INJURY AND FUTURE DISEASE RISK
}

\author{
Mina Lateva ${ }^{1}$, Dimitrichka Bliznakova ${ }^{1}$, Sonya Galcheva ${ }^{1}$, Yana Bocheva ${ }^{2}$, \\ Maria Neshkinska ${ }^{1}$, Vilhelm Mladenov ${ }^{3}$, Irina Halvadzhiyan ${ }^{1}$, Galina Yordanova ${ }^{1}$, \\ Veselin Boyadzhiev ${ }^{1}$, Yoto Yotov $^{4}$, Nikolay Conev ${ }^{5}$, Violeta Iotova ${ }^{1}$ \\ ${ }^{1}$ Department of Pediatrics, Faculty of Medicine, Medical University of Varna \\ ${ }^{2}$ Department of General Medicine and Clinical Laboratory, Faculty of Medicine, \\ Medical University of Varna \\ ${ }^{3}$ First Pediatric Clinic with a Pediatric ICU, St. Marina University Hospital, Varna \\ ${ }^{4}$ Department of Internal Diseases, Faculty of Medicine, Medical University of Varna \\ ${ }^{5}$ Department of Propedeutics of Internal Diseases, Faculty of Medicine, \\ Medical University of Varna
}

\begin{abstract}
INTRODUCTION: Evidence associates obesity with glomerular hyperfiltration. Concurrent inflammation, hypertension, dyslipidemia, and insulin resistance represent further established risks to renal health in both children and adults. Our aim was to investigate the relationship between childhood obesity and the risk of renal impairment.

MATERIALS AND METHODS: A total of 114 obese but otherwise healthy children, who were investigated in 2013, were included in the study (mean age 11.5 \pm 3.6 ). Auxology was measured using standard procedures. The pubertal status was defined applying the Tanner scale. Blood and urine tests were performed after overnight fast. Kidney size was measured by standard abdominal ultrasound; kidney volume and estimated glomerular filtration rate were calculated.

RESULTS: Microalbuminuria was detected in $8.5 \%$ of the children, and $40.2 \%$ of all patients were with high systolic blood pressure (BP). Close to half of the children (43.4\%) showed insulin resistance (assessed by HOMA-IR) and $95.4 \%$ displayed hyperinsulinism. Kidney volume correlated with waist circumference (WC) (left $r=0.636, p<0.001$; right $r=0.532, p<0.001$ ), as well as with weight and elevated systolic BP. Children with hyperfiltration (9.6\% of all) at this stage all had elevated total cholesterol and triglycerides. In further $14.3 \%$ of the children (all pubertal) the glomerular filtration rate was low, and they had significantly larger kidney volume (left $p<0.001$; right $p=0.004)$, HOMA-IR $(p=0.001)$, WC and BMI $(p<0.001)$ compared to those with normal and hyperfiltration rate.
\end{abstract}

Address for correspondence:

Mina Lateva

Department of Pediatrics

Faculty of Medicine

Medical University of Varna

55 Marin Drinov St.

9002 Varna

e-mail:mina_pl@yahoo.com

Received: February 21, 2017

Accepted: March 31, 2017
CONCLUSION: To summarize, childhood obesity showed an association with established risk factors for renal toxicity. Whether kidney volume in obese children has an independent predictive value of future chronic kidney disease remains to be investigated.

Keywords: childhood obesity, microalbuminuria, glomerular filtration, kidney volume 
Mina Lateva, Dimitrichka Bliznakova, Sonya Galcheva et al.

\section{INTRODUCTION}

The prevalence of childhood obesity has doubled and tripled in the developed (1) and developing (2) countries during the last decade. Although there is a reported stabilization in the trends of obesity in developed countries such as USA (3), the prevalence across the World and Europe remains high $(4,5)$.

Obesity has long-term adverse effects on morbidity and mortality. It is associated with cardiovascular, liver, kidney, and metabolic disorders in adulthood (6). Since metabolic and cardiovascular complications may be present in early childhood and adolescence, renal risk has also been investigated. Along with childhood obesity, the prevalence of endstage renal disease (ESRD) has also drastically increased (7). Several studies indicate that childhood obesity is associated with an increased risk of kidney disease and the progression and mortality of CKD (8). Becoming overweight at a younger age is associated with higher odds of developing chronic kidney disease (CKD) by age 60-64 years (9). Whether it is about the timing of onset or the duration of obesity, further investigations are needed, as consequences of childhood obesity on kidney disease and risk data are very limited.

Obesity and hypertension both lead to cardiovascular complications. In addition, obesity per se can promote hypertension (10). In the early stage of obesity in adults, renal hemodynamics is characterized by renal hypercirculation and glomerular hyperfiltration, particularly in the presence of hypertension (11). Pathogenetically, numerous factors are involved, e.g. enhanced glomerular capillary pressure, adrenergic nerve overactivity, inappropriate activation of the renin-angiotensin-aldosterone system, insulin resistance, hyperinsulinemia, dyslipidemia, enhanced clotting tendency and sodium retention (12). Kidney damage is clinically manifested as loss of albumin in the urine or decline in the glomerular filtration rate (GFR). Analysis of NHANES data showed that abdominal obesity in adults was associated with both a decrease in GFR and microalbuminuria (13).

Originally, microalbuminuria (MA) was introduced as a biomarker of early diabetic nephropathy (14); large epidemiological trials have indicated it is a powerful and independent predictor of all-cause mortality and endothelial dysfunction in diabetic and non-diabetic adults $(15,16)$. Most studies suggest MA is the result of increased vascular leakage as a reflection of general vascular damage (17). Albuminuria as a more potent sign of endothelial dysfunction is an early marker of CKD and is a target of primary prevention measures (18). However, the role of elevated albumin excretion rate as an early marker of kidney disease risk in children has not been thoroughly studied. Sanad et al. (19) found that BMI and abdominal obesity significantly increased the odds of microalbuminuria. Csernus et al. (20) found in their study that clinically healthy obese children had a higher degree of albuminuria than normal-weight children, indicating early renal glomerular and tubular dysfunction as part of the morbidity of childhood obesity. There are also studies on children and adolescents reporting controversial results. Hirschler et al. report that overweight/obesity (OW/OB) is inversely associated with childhood albuminuria (21) and Rademacher et al. report that albumin excretion rate in adolescence is not significantly related to age, body size (BMI), insulin resistance, or the cardiovascular risk factors associated with the insulin resistance syndrome (BMI, systolic BP, triglycerides, HDL-C)(22). Whether obesity in youth uniformly affects the presence of MA and whether variations in albumin excretion are related to other renal risk factors remains to be determined.

The aim of this study was to investigate the relationship between childhood overall and abdominal obesity and risk of renal impairment.

\section{MATERIALS AND METHODS}

The study was conducted in the St. Marina University Hospital, Varna, Bulgaria during a one-year period (Nov, 2012-Nov, 2013). Children included in the study were consecutively admitted to the pediatric clinic for diagnostics and formulation of the treatment strategy of obesity. Parental consent for all the procedures was obtained. Weight and height were measured according to standard procedures (23) with SECA 861 weight scale and Harpenden wallmounted stadiometer. Waist circumference (WC) to define abdominal obesity was measured with SECA 201 tape at the middle of the medial axillary line connecting the $10^{\text {th }}$ rib and crista iliaca (24). Body mass index (BMI) was calculated using height and weight 
data according to a standard formula - BMI=weight $(\mathrm{kg}) /$ height $^{2}\left(\mathrm{~m}^{2}\right)$. Children defined as obese according to the CDC growth references were invited to participate (25). Exclusion criteria included acute infection, fever of unknown origin, kidney disease, type 1 diabetes mellitus and refusal to participate. The patients' pubertal status was defined according to Tanner scale. Blood pressure was measured according to standard procedures. Kidney size was measured by standard abdominal ultrasound using ALOKA - Prosound SSD-4000SV. Kidney volume was calculated using the following formula - length $\mathrm{x}$ width $\mathrm{x}$ anterior posterior diameter $\mathrm{x} 0.523$ (26). Glomerular filtration rate was estimated (eGFR) using the Schwartz formula, adjusted to $\mathrm{ml} / \mathrm{min} \cdot 1.73 \mathrm{~m}^{2}$ and levels of normal, hyperfiltration and low filtration rate according to sex and age were used (27).

Children and their parents were interviewed for obesity-related complaints (snoring, being tired after a mild physical activity, joint pain and enuresis) as well as for family history of obesity, diabetes, hypertension, any chronic diseases.

\section{Sample Collection}

A fasting venous blood sample was collected in the morning for determining of fasting blood glucose level (BGL), serum insulin, lipid profile, high-sensitivity C-reactive protein (hsCRP), serum albumin, urea and creatinine levels. An oral glucose tolerance test (OGTT) was performed with glucose load equivalent to $75 \mathrm{~g}$ of glucose powder solved in water or 1.75 $\mathrm{g}$ x weight, not exceeding a total of $75 \mathrm{~g}$. Blood samples were collected before taking the glucose and at 30 min of OGTT for determining BGL and serum insulin, as well as at $60 \mathrm{~min}$ and $120 \mathrm{~min}$ for BGL only.

Morning urine sample was collected, after the child and his/her parents were instructed to avoid excessive physical activity. Urine analysis was performed to determine microalbuminuria (MA).

\section{Laboratory Analyses}

Blood glucose levels were determined with the hexokinase method using biochemical analyzer Olympus AU 400. Serum insulin levels were determined using immunological analyzer IMMULITE 2000. Levels of serum lipids - total cholesterol, triglycerides, HDL-cholesterol, LDL-cholesterol, serum levels of albumin, urea and creatinine were analyzed using automatic biochemical analyzer Olympus AU 400 .

Homeostasis model assessment for insulin resistance (HOMA-IR) was used to calculate and define insulin sensitivity and resistance according to the formula: fasting blood glucose $(\mathrm{mmol} / \mathrm{l}) \mathrm{x}$ fasting serum insulin $(\mu \mathrm{IU} / \mathrm{ml}) / 22.5$. Insulin resistance was defined as a HOMA-IR score > 3.5 (27), while hyperinsulinism was defined as fasting serum insulin levels $>29.1 \mu \mathrm{IU} / \mathrm{ml}$.

Impaired fasting glucose was defined as plasma glucose levels between 5.6 and $6.9 \mathrm{mmol} / \mathrm{l}$, impaired glucose tolerance was defined as two hours post load glucose plasma levels between 7.8 and $11.1 \mathrm{mmol} / \mathrm{l}$.

Elevated total cholesterol, triglycerides, LDLcholesterol, hsCRP, urea and creatinine were defined according to laboratory references. Low HDL-cholesterol and serum albumin were defined likewise.

Microalbuminuria was defined as urine albumin ranging from 30 to $300 \mathrm{mg}$ from a morning spot urine sample estimated for 24-hour urine collection.

The presence of hypertension (systolic, diastolic or both) was defined according to NHBPEP (2004) as a measure above $95^{\text {th }}$ percentile for age, sex and height (29).

\section{Statistical Analysis}

Statistical analysis was performed using SPSS for Windows ver. 19. Descriptive analysis was applied. Logistic regression models were used to analyze associations with MA, kidney volume and estimated glomerular filtration. Relations between the latter and laboratory data, family history and weight status were analyzed using correlation analysis. Statistically significant differences were accepted if $\mathrm{p} \leq 0.05$.

\section{RESULTS}

The mean age of the group of 114 children and adolescents was $11.5 \pm 3.6$ years (median 10.6, age range from 4 to 17.6 years), $38.6 \%$ boys. The mean BMI z-score was $2.14 \pm 0.42$. The children in a prepubertal stage (Tanner I) were $38.1 \%$ (48.8\% boys, $\mathrm{n}=21$ ). With high systolic BP were $40.2 \%$. Impaired fasting glucose was found in $29.8 \%$ of all and $13.4 \%$ of the children were with impaired glucose tolerance. Almost half of the children (43.4\%) showed insulin resistance and $95.4 \%$ displayed hyperinsulinism. El- 
Mina Lateva, Dimitrichka Bliznakova, Sonya Galcheva et al.

evated hsCRP was present in $32.5 \%$. Elevated LDLcholesterol was found in $42.1 \%$ of the children. Lower HDL cholesterol had $21.9 \%$ and with high total cholesterol were $6.2 \%$ of all.

Microalbuminuria was detected in $8.5 \%(n=9)$ of the children, the parents of whom had all reported a family history of arterial hypertension. The following analysis was stratified according to the presence or absence of MA. We found no significant differences in sex, BMI, WC between the two groups, including other obesity-related signs of current morbidity e.g. joint pain, easy fatigue reported by the children (see Table 1). In contrast, there were marked differences in both insulin resistance and systolic BP between the two groups. As shown in Table 1, there was no significant difference between the groups in terms of elevated systolic BP. This can be contributed to the relatively high percentage (40.2\%) of children with elevated systolic BP.
To further evaluate MA in relation to pubertyassociated changes in metabolic risk factors we compared the complication factors among three subgroups: Group 1 - children staged Tanner I - prepubertal; Group 2 - children staged Tanner II and III; and Group 3 were staged Tanner IV and V. There were no significant differences between the groups in terms of MA and all studied metabolic parameters, except for fasting insulin and insulin resistance. Children in the $1^{\text {st }}$ group (prepubertal) had significantly lower fasting insulin compared to the $3^{\text {rd }}$ group (mean $12.83 \pm 7.49$ vs. $19.18 \pm 10.19, \mathrm{p}=0.011$ ) and the prevalence of insulin resistance in the $1^{\text {st }}$ group was significantly lower than that of the $3^{\text {rd }}$ group (30.2\% vs. $61 \%, \mathrm{p}=0.013)$.

After calculating eGFR for age and sex we stratified the children accordingly - children with normal eGFR, children with hyperfiltration rate and children with low eGFR. Those with hyperfiltration were $9.6 \%$ of all and they all had elevated total cho-

Table 1. Comparison of different variables between the MAU groups

\begin{tabular}{lccc}
\hline & MAU (-) & MAU (+) & Sig. \\
Sex (male/female \%) & $58.8 / 41.2$ & $22.2 / 78.82 / 7$ & 0.269 \\
BMI z-score & $2.15 \pm 0.42$ & $2.22 \pm 0.34$ & 0.633 \\
WC, cm & $91.14 \pm 15.21$ & $90.53 \pm 9.08$ & 0.907 \\
Systolic BP, mmHg & $111.3 \pm 13.7$ & $123.3 \pm 19.4$ & $\mathbf{0 . 0 4 5}$ \\
Diastolic BP, mmHg & $70.0 \pm 10.3$ & $76.7 \pm 12.1$ & 0.135 \\
Fasting blood glucose, mmol/1 & $5.16 \pm 0.69$ & $5.23 \pm 0.48$ & 0.761 \\
Fasting insulin, $\mu$ IU/ml & $16.1 \pm 10.4$ & $16.3 \pm 3.8$ & 0.953 \\
Insulin resistance (HOMA-IR), \% & 37.5 & 88.9 & $\mathbf{0 . 0 0 3}$ \\
Elevated total cholesterol, \% & 7.3 & 0 & 0.407 \\
Elevated triglycerides, \% & 1.0 & 0 & 0.762 \\
Low HDL-cholesterol, \% & 21.6 & 33.3 & 0.944 \\
Elevated LDL-cholesterol, \% & 40.2 & 55.6 & 0.376 \\
Elevated CRP, \% & 30.9 & 55.6 & 0.135 \\
Hyperinsulinism, \% & 95.7 & 100 & 0.528 \\
Joint pain, \% & 26.8 & 11.1 & 0.306 \\
Easy fatigue, \% & 47.9 & 33.3 & 0.407 \\
\hline
\end{tabular}


Table 2. Comparison of different variables between the eGFR groups (ANOVA)

\begin{tabular}{|c|c|c|c|c|c|}
\hline \multirow{2}{*}{$\begin{array}{l}\text { Dependent } \\
\text { Variable }\end{array}$} & \multirow{2}{*}{ (I) } & \multirow{2}{*}{ (J) } & \multirow{2}{*}{ Sig. } & \multicolumn{2}{|c|}{ 95\% Confidence Interval } \\
\hline & & & & Lower Bound & Upper Bound \\
\hline \multirow{6}{*}{ BMI z_score } & \multirow{2}{*}{ low filtration rate } & normal & 1.000 & -0.19 & 0.36 \\
\hline & & hyperfiltration & 0.206 & -0.09 & 0.69 \\
\hline & \multirow{2}{*}{ normal } & low filtration rate & 1.000 & -0.36 & 0.19 \\
\hline & & hyperfiltration & 0.319 & -0.11 & 0.53 \\
\hline & \multirow{2}{*}{ hyperfiltration } & low filtration rate & 0.206 & -0.69 & 0.09 \\
\hline & & normal & 0.319 & -0.54 & 0.11 \\
\hline \multirow{6}{*}{ WC } & \multirow{2}{*}{ low filtration rate } & normal & 0.000 & 9.32 & 27.01 \\
\hline & & hyperfiltration & 0.000 & 13.19 & 38.45 \\
\hline & \multirow{2}{*}{ normal } & low filtration rate & 0.000 & -27.01 & -9.32 \\
\hline & & hyperfiltration & 0.227 & -2.73 & 18.04 \\
\hline & \multirow{2}{*}{ hyperfiltration } & low filtration rate & 0.000 & -38.45 & -13.19 \\
\hline & & normal & 0.227 & -18.04 & 2.73 \\
\hline \multirow{6}{*}{$\begin{array}{l}\text { fasting blood } \\
\text { glucose }\end{array}$} & \multirow{2}{*}{ low filtration rate } & normal & 0.838 & -0.24 & 0.64 \\
\hline & & hyperfiltration & 1.000 & $-0,52$ & 0.75 \\
\hline & \multirow{2}{*}{ normal } & low filtration rate & 0.838 & -0.64 & 0.24 \\
\hline & & hyperfiltration & 1.000 & -0.59 & 0.44 \\
\hline & \multirow{2}{*}{ hyperfiltration } & low filtration rate & 1.000 & -0.75 & 0.52 \\
\hline & & normal & 1.000 & -0.44 & 0.59 \\
\hline \multirow{6}{*}{$\begin{array}{l}\text { fasting serum } \\
\text { insulin }\end{array}$} & \multirow{2}{*}{ low filtration rate } & normal & 0.007 & 1.76 & 14.29 \\
\hline & & hyperfiltration & 0.000 & 6.06 & 24.07 \\
\hline & \multirow{2}{*}{ normal } & low filtration rate & 0.007 & -14.29 & -1.76 \\
\hline & & hyperfiltration & 0.066 & -0.33 & 14.40 \\
\hline & \multirow{2}{*}{ hyperfiltration } & low filtration rate & 0.000 & -24.07 & -6.06 \\
\hline & & normal & 0.066 & -14.40 & 0.33 \\
\hline \multirow{6}{*}{ HOMA_IR } & \multirow{2}{*}{ low filtration rate } & normal & 0.007 & 0.47 & 3.81 \\
\hline & & hyperfiltration & 0.001 & 1.27 & 6.06 \\
\hline & \multirow{2}{*}{ normal } & low filtration rate & 0.007 & -3.81 & -0.47 \\
\hline & & hyperfiltration & 0.184 & -0.44 & 3.49 \\
\hline & hyperfiltration & low filtration rate & 0.001 & -6.06 & -1.27 \\
\hline & пуретіпtration & normal & 0.184 & -3.49 & 0.44 \\
\hline & low filtration rate & normal & 1.000 & -0.17 & 0.15 \\
\hline & low illtration rate & hyperfiltration & 1.000 & -0.17 & 0.29 \\
\hline elevated total & normol & low filtration rate & 1.000 & -0.15 & 0.17 \\
\hline cholesterol & Hormal & hyperfiltration & 1.000 & -0.12 & 0.26 \\
\hline & hynerfiltration & low filtration rate & 1.000 & -0.29 & 0.17 \\
\hline & 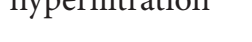 & normal & 1.000 & -0.27 & 0.12 \\
\hline & low filtration rate & normal & 0.045 & 0.001 & 0.12 \\
\hline & How intidation Iate & hyperfiltration & 0.264 & -0.03 & 0.15 \\
\hline elevated TG & normal & low filtration rate & 0.045 & -0.12 & -0.001 \\
\hline & & hyperfiltration & 1.000 & -0.07 & 0.07 \\
\hline & honon & low filtration rate & 0.264 & -0.15 & 0.03 \\
\hline & 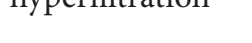 & normal & 1.000 & -0.07 & 0.07 \\
\hline
\end{tabular}


Mina Lateva, Dimitrichka Bliznakova, Sonya Galcheva et al.

\begin{tabular}{|c|c|c|c|c|c|}
\hline \multirow{6}{*}{$\begin{array}{l}\text { low HDL- } \\
\text { cholesterol }\end{array}$} & \multirow{2}{*}{ low filtration rate } & normal & 0.429 & -0.14 & 0.57 \\
\hline & & hyperfiltration & 0.502 & -0.79 & 0.22 \\
\hline & \multirow{2}{*}{ normal } & low filtration rate & 0.429 & -0.57 & 0.14 \\
\hline & & hyperfiltration & 0.012 & -0.92 & -0.09 \\
\hline & \multirow{2}{*}{ hyperfiltration } & low filtration rate & 0.502 & -0.22 & 0.79 \\
\hline & & normal & 0.012 & 0.09 & 0.92 \\
\hline \multirow{6}{*}{$\begin{array}{l}\text { elevated LDL- } \\
\text { cholesterol }\end{array}$} & \multirow{2}{*}{ low filtration rate } & normal & 1.000 & -0.23 & 0.43 \\
\hline & & hyperfiltration & 1.000 & -0.43 & 0.52 \\
\hline & \multirow{2}{*}{ normal } & low filtration rate & 1.000 & -0.43 & 0.23 \\
\hline & & hyperfiltration & 1.000 & -0.44 & 0.33 \\
\hline & \multirow{2}{*}{ hyperfiltration } & low filtration rate & 1.000 & -0.52 & 0.43 \\
\hline & & normal & 1.000 & -0.33 & 0.44 \\
\hline
\end{tabular}

lesterol and triglycerides. In $14.3 \%$ of the children (all pubertal) there was low glomerular filtration rate, and they had significantly larger kidney volume (left $\mathrm{p}<0.001$; right $\mathrm{p}=0.004)$, HOMA-IR $(\mathrm{p}=0.001)$ compared to the other children. There was a significant difference in WC when comparing the eGFR groups (ANOVA, see Table 2), the group with low eGFR had significantly larger WC compared to the group with hyperfiltration and even larger when compared to normal eGFR children $(\mathrm{p}<0.001)$. There was a significant difference in fasting insulin $(\mathrm{p}<0.001)$ and HOMA-IR ( $\mathrm{p}=0.001)$. All blood glucose values during the OGTT had no significant differences across the eGFR groups, including fasting glucose.

Kidney volume correlated with WC (left $\mathrm{r}=0.656, \mathrm{p}<0.001$; right $\mathrm{r}=0.532, \mathrm{p}<0.001$ ), as well as with weight (left $\mathrm{r}=0.732, \mathrm{p}<0.001$; right $\mathrm{r}=0.576$, $\mathrm{p}<0.001$ ) and elevated systolic BP (left $\mathrm{r}=0.478$, $\mathrm{p}<0.001$; right $\mathrm{r}=0.362, \mathrm{p}=0.001)$. Stratification according to eGFR (see above) showed that children with low filtration rate had the largest left kidney volume compared to those with hyper and normal filtration rate (resp. $\mathrm{p}<0.001 ; \mathrm{p}=0.001$ ). The difference in the left kidney volume between the group with hyperfiltration and normal eGFR was marginally significant ( $\mathrm{p}=0.071)$. Significant difference in the right kidney volume with the same direction was registered only comparing the children with low and normal eGFR (p=0.006).

\section{DISCUSSION}

In this study we assessed the presence of a modest elevation of MA in a cohort of obese, but otherwise healthy children and adolescents. Consistent with the prevalence of about $10 \%$ in obese adolescents (30), we found that $8.5 \%$ of the obese children in our group had MA. Another more recent study of Lurbe et al. (31) identified in Spain a prevalence of $2.4 \%$ of MA in obese youths thus suggesting the existence of important confounding variables, e.g. orthostatic proteinuria.

The presence of MA in adults predicts CV morbidity/mortality in otherwise healthy individuals (15). While there is a general consensus on the fact that MA is a marker for systemic endothelial dysfunction and preclinical atherosclerosis, it remains controversial as to which of the potential vascular toxins gives rise to MA. Concluding that MA is quite common in obese children and adolescents, we were interested in determining how it relates to other risk factors in this young group. The major findings of our study were the strong positive correlation between the presence of MA and fasting insulin and insulin resistance. Studies in adults with type 2 diabetes have demonstrated that insulin resistance may contribute to micro- and macrovascular diseases (32). Being overweight/obese is the most important risk factor for the development of T2DM, even in youths. In a group of Japanese subjects with type 2 diabetes, insulin resistance was associated with MA and direct measures of endothelial function (33). The physiological decrease in insulin sensitivity associated with normal pubertal development is further exacerbated by the presence of obesity. As hyperinsulinemia influences blood pressure and serum lipids, it often results in hypertension and dyslipidemia. Therefore, metabolic consequences of obesity and not obesity per se may be the driving forces behind 
urinary albumin excretion, and even slight abnormalities in glucose metabolism below the diagnostic cutoff for diabetes contributed to the presence of microalbuminuria (34). MA has a strong positive relation to post-challenge alterations in glucose metabolism and overall loss of insulin sensitivity.

Multiple mechanisms have postulated that obesity directly impacts kidney disease including hyperfiltration, increased glomerular capillary wall tension, and podocyte stress. Apart from adiposity itself, the high caloric intake that leads to obesity may also increase chronic kidney disease risk (35). GFR is the most useful indicator of kidney function and kidney disease progression. However, determination of true GFR is difficult to perform for regular clinical use in children, thus formulas were developed to estimate GFR using endogenous surrogate markers such as creatinine (27). Studies have shown that obesity is associated with elevated GFR and increased renal blood flow. These effects may contribute to hyperfiltration, glomerulomegaly, and later focal glomerulosclerosis (36). Overweight and obesity are strongly associated with numerous cardiovascular disease risk factors like hyperinsulinemia, impaired glucose metabolism, hypertension, hyperlipidemia and metabolic syndrome, which, apart from predisposing to cardiovascular disease, are also independent risk factors for CKD. Reduced insulin sensitivity represents the most important link between obesity and other metabolic complications leading to renal injury (34). Several studies give strong evidence that obesity, in particular central body fat distribution, is important for renal function abnormalities. The pattern of obesity may affect renal hemodynamics and an elevated BMI with central obesity results in reduced GFR and reduced effective renal blood flow as opposed to obesity with peripheral fat distribution. In a study by Iseki et al. (37), conducted in 1,572 young men, metabolic risk factors and renal function were investigated. These investigators reported that metabolic risk clustering of three or more risk factors was strongly associated with glomerular hyperfiltration. This early renal functional abnormality was especially associated with adiposity and elevated blood pressure. Based on these data, the authors proposed that increased adiposity, particularly visceral fat production of inflammatory adipokines, was a major driving force of glomerular hyperfiltra- tion. There are similar studies in children confirming that visceral fat tissue in children might be a predictor for the metabolic syndrome (38). The obesityrelated early renal functional changes may be especially detrimental in populations manifesting lower birth weight and lower nephron numbers (39). Bagby (40) suggested that intrauterine growth restriction may lead to decreased number of nephrons and impaired kidney development, which if combined with an excessive infant "catchup" growth after birth may result in a mismatch between body size and nephron number. This would predispose to nephron hyperfiltration and hypertension later in life. Other factors, such as systemic and renal inflammation and oxidative stress, may determine to what extent this hyperfiltration leads to glomerular and tubulointerstitial fibrosis and progressive renal disease.

\section{CONCLUSION}

Obesity affects metabolic equilibrium before adulthood. There is clear evidence that, in adults, excess body weight is significantly associated with an increased risk of kidney disease. A similar association has also been documented in obese children and adolescents, even though there is a lack of large and long-term studies. To summarize, childhood obesity showed association with established risk factors for renal toxicity. The current findings suggest development of kidney dysfunction in obese children with positive family history of hypertension after the initiation of puberty. Microalbuminuria might be a good marker to identify pubertal children with increased risk of endovascular damage. Whether kidney volume in obese children has an independent predictive value of future kidney disease remains to be investigated.

\section{REFERENCES}

1. Ogden CL, Carroll MD, Curtin LR, McDowell MA, Tabak CJ, Flegal KM. Prevalence of overweight and obesity in the United States, 1999-2004. JAMA 2006; 295(13):1549-55.

2. Iotova V, Galcheva S, Petrova K. Obesity is increasing fast among Varna school children in the last 5 years (2002-2007). Scripta Scientifica Medica 2009, 41(1): 31-34

3. Ogden CL, Carroll MD, Kit BK, Flegal KM. Prevalence of childhood and adult obesity in the United 
Mina Lateva, Dimitrichka Bliznakova, Sonya Galcheva et al.

States, 2011-2012. JAMA 2014; 311(8):806-14. doi: 10.1001/jama.2014.732.

4. WHO. Childhood overweight and obesity. Fact sheet No 311, March 2011. http://www.who.int/mediacentre/factsheets/fs311/en/index.html

5. Van Stralen MM, te Velde SJ, van Nassau F, Brug J, Grammatikaki E, Maes L, De Bourdeaudhuij I, Verbestel V, Galcheva S, Iotova V, Koletzko BV, von Kries R, Bayer O, Kulaga Z, Serra-Majem L, Sánchez-Villegas A, Ribas-Barba L, Manios Y, Chinapaw MJ; ToyBox-study group. Weight status of European preschool children and associations with family demographics and energy balance-related behaviours: a pooled analysis of six European studies. Obes Rev 2012; 13 Suppl. 1:29-41. doi: 10.1111/j.1467-789X.2011.00959.x.

6. Kopelman P. Obesity as a medical problem. Nature 2000;. 404(6778):635-43.

7. USRDS: The United States Renal Data System (2003) Am J Kidney Dis 2003; 42:1-230.

8. Wang Y, Chen X, Klag MJ, Caballero B. Epidemic of childhood obesity: implications for kidney disease. Adv Chronic Kidney Dis 2006; 13(4):336-51.

9. Silverwood RJ, Pierce M, Thomas C, Hardy R, Ferro C, Sattar N, Whincup P, Savage C, Kuh D, Nitsch D and on behalf of the National Survey of Health and Development Scientific and Data Collection Teams. Association between younger age when first overweight and increased risk for CKD. J Am Soc Nephrol 2013; 24(5):813-21. doi: 10.1681/ ASN.2012070675.

10. Antic V, Dulloo A, Montani JP. Multiple mechanisms involved in obesity-induced hypertension. Heart Lung Circ 2003; 12(2):84-93.

11. Srivastava T. Nondiabetic consequences of obesity on kidney. Pediatr Nephrol 2006; 21(4):463-70. Epub 2006 Feb 21.

12. Adelman RD. Obesity and renal disease. Curr Opin Nephrol Hypertens 2002; 11(3):331-5.

13. Chen J, Muntner P, Hamm LL, Jones DW, Batuman V, Fonseca V, Whelton PK, He J. The metabolic syndrome and chronic kidney disease in U.S. adults. Ann Intern Med 2004;140:167-174.

14. Mogensen CE. Microalbuminuria predicts clinical proteinuria and early mortality in maturity-onset diabetes. N Engl J Med 1984; 310: 356-360.

15. Gerstein HC, Mann JFE, Yi Q, Zinman B, Dinneen SF, Hoogwerf B, Halle JP, Young J, Rashkwo A,
Joyce C, Nawaz S, Yusuf S; for the HOPE Study Investigators. Albuminuria and risk of cardiovascular events, death, and heart failure in diabetic and nondiabetic individuals. JAMA 2011; 286:421-426.

16. Chang A, Van Horn L, Jacobs DR Jr, Liu K, Muntner P, Newsome B, Shoham DA, Durazo-Arvizu R, Bibbins-Domingo K, Reis J, Kramer H. Lifestyle-related factors, obesity, and incident microalbuminuria: the CARDIA (Coronary Artery Risk Development in Young Adults) study. Am J Kidney Dis 2013; 62(2):267-75. doi: 10.1053/j.ajkd.2013.02.363.

17. Pedrinelli R, Dell'Omo G, Penno G, Mariani M. Non-diabetic microalbuminuria, endothelial dysfunction and cardiovascular disease. Vasc Med 2001; 6:257-264.

18. Feldt-Rasmussen B. Microalbuminuria, endothelial dysfunction and cardiovascular risk. Diabetes Metab 2000; 26 (Suppl 4): 64-66.

19. Sanad M, Gharib A. Evaluation of microalbuminuria in obese children and its relation to metabolic syndrome. Pediatr Nephrol 2011; 26(12):2193-9. doi: 10.1007/s00467-011-1931-9. Epub 2011 Jun 3.

20. Csernus K, Lanyi E, Erhardt E, Molnar D. Effect of childhood obesity and obesity-related cardiovascular risk factors on glomerular and tubular protein excretion. Eur J Pediatr 2005; 164: 44-49.

21. Hirschler V, Molinari C, Maccallini G, Aranda C. Is albuminuria associated with obesity in school children? Pediatr Diabetes 2010; 11(5):322-30. doi: 10.1111/j.1399-5448.2009.00599.x.

22. Rademacher E, Mauer M, Jacobs DR, Jr, Chavers B, Steinke J, and Sinaiko A. Albumin Excretion Rate in Normal Adolescents: Relation to Insulin Resistance and Cardiovascular Risk Factors and Comparisons to Type 1 Diabetes Mellitus Patients. Clin J Am Soc Nephrol 2008; 3(4): 998-1005. doi:10.2215/CJN.04631007 PMCID: PMC2440272

23. Galcheva S, Iotova V, Petrova K, Stratev V, Tzaneva V. Incidence of obesity and overweight among children and adolescents from Varna. Pediatria 2009; 4: $15-18$

24. Galcheva S, Iotova V, Yotov Y, Grozdeva K, Stratev V, Tzaneva V. Waist circumference percentile curves for Bulgarian children and adolescents aged 6-18 years. Int J Pediatr Obes 2009; 4(4):381-8. doi: $10.3109 / 17477160902846195$.

25. Kuczmarski RJ, Ogden CL, Grummer-Strawn LM, Flegal KM, Guo SS, Wei R, Mei Z, Curtin LR, Roche AF, Johnson CL (2000) CDC growth charts: 
United States. National Center for Health Statistics, Hyattsville

26. Dinkel E, Ertel M, and Dittrich M. Kidney size in childhood. Sonographical growth charts for kidney length and volume. Pediatric Radiology 1985, vol. 15 , no. 1 , pp. $38-43$.

27. Schwartz George J and Work Dana F. Measurement and Estimation of GFR in Children and Adolescents. Clin J Am Soc Nephrol 2009; 4: 1832-1843.

28. Önal Z, Atasayan V, Akici N, Gürbüz T, Nuhoğlu Ç. The Relationship between Glycosylated Haemoglobin (HbA1c) Levels and Serum Lipid Profiles in Insulin Resistant Children HK J Paediatr 2014; 19:22-27

29. The fourth report on the Diagnosis, Evaluation, and Treatment of High Blood Pressure in Children and Adolescents. NIH Publication No. 05-5267; Originally printed September 1996 (96-3790), Revised May 2005.

30. Burgert TS, Dziura J, Yeckel C, Taksali SE, Weiss $\mathrm{R}$, Tamborlane W, Caprio S. Microalbuminuria in pediatric obesity: prevalence and relation to other cardiovascular risk factors. Int J Obes (Lond) 2006; 30: 273-280.

31. Lurbe E, Torro MI, Alvarez J, Aguilar F, Fernandez-Formoso JA, Redon J. Prevalence and factors related to urinary albumin excretion in obese youths. J Hypertens 2013; 31(11):2230-6; discussion 2236. doi: 10.1097/HJH.0b013e328364bcbf.

32. Vedovato M, Lepore G, Coracina A, Dodesini AR, Jori E, Tiengo A, Del Prato S, Trevisan R. Effect of sodium intake on blood pressure and albuminuria in type 2 diabetic patients: the role of insulin resistance. Diabetologia 2004; 47: 300-303.

33. Yu Y, Suo L, Yu H, Wang C, Tang H. Insulin resistance and endothelial dysfunction in type 2 diabetes patients with or without microalbuminuria. Diabetes Res Clin Pract 2004; 65: 95-104.

34. Savino A, Pelliccia P, Chiarelli F, Mohn A (2010) Obesity-Related Renal Injury in Childhood. Horm Res Paediatr; 73:303-311 (DOI:10.1159/000308161).

35. Wickman C, Kramer H. Obesity and kidney disease: potential mechanisms. Semin Nephrol 2013; 33(1):14-22. doi: 0.1016/j.semnephrol.2012.12.006.

36. Ihab M, Wahba and Robert H. Mak Obesity and Obesity-Initiated Metabolic Syndrome: Mechanistic Links to Chronic Kidney Disease. Clin J Am Soc Nephrol 2007; 2: 550-562, 2007. doi: 10.2215/ CJN.04071206.
37. Iseki K, Ikemiya $\mathrm{Y}$, Kinjo $\mathrm{K}$, Inoue $\mathrm{T}$, Iseki $\mathrm{C}$ and Takishita S. Body mass index and the risk of development of end-stage renal disease in a screened cohort. Kidney Int 2004; 65: 1870-1876.

38. McCarthy HD. Body fat measurements in children as predictors for the metabolic syndrome: focus on waist circumference. Proceedings of the Nutrition Society 2006; 65 385-392.

39. White SL, Perkovic V, Cass A, Chang CL, Poulter NR, Spector T, Haysom L, Craig JC, Salmi IA, Chadban SJ, Huxley RR. Is low birth weight an antecedent of CKD in later life? A systematic review of observational studies. Am J Kidney Dis 2009; 54 : 248-261.

40. Bagby SP. Obesity-initiated metabolic syndrome and the kidney: A recipe for chronic kidney disease? J Am Soc Nephrol 2004; 15: 2775-2791. 